\title{
An image compression using using discrete cosine transforms and JPEG enconder
}

\author{
Ravi Mathey ${ }^{1}$, Prof.P.S.Avadhani ${ }^{2}$ \\ ${ }^{1}$ Associate Professor and HOD of CSE Department, Vidya Jyothi Institute of Technology. \\ ${ }^{2}$ Professor, Department Of Computer Science \& Systems Engineering, College Of Engineering Andhra \\ University, Visakhapatnam ,India
}

\begin{abstract}
The proposed Multiresolution decomposition through wavelets transform of fractals coded images reveal strong relationships limit the frequency content. The research is includes other extensions of the wavelet transform and fractals are wavelet transform. And our work deals with deals with the JPEG Encoder for image compression upon comparing with Discrete cosine transform (DCT) is widely used in image processing, especially for compression. Some of the applications of two-dimensional DCT involve still image compression and compression of individual video frames.The JPEG standard has been an effective first solution to the standardization of image and the performance of DWT compression with DCT compression. And JPEG 2000 is a wavelet-based standard for the compression of still digital images The wavelet transform has emerged as a cutting edge technology, within the field of image compression. And Wavelet- based coding provides substantial improvements in picture quality at higher compression ratios.and also dicuss about of the proposed DCT based JPEG Encoder and Wavelet based JPEG Encoder.
\end{abstract}

Keywords: Multi resolution, image compression, DCT, JPEG 2000 Wavlet standard.

\section{Introduction}

Wavelets have generated a tremendous interest in both theoretical and applied areas, especially over the past few years. The number of researchers, already large, continues to grow, so progress is being made at a rapid pace. In fact, advancements in the area are occurring at such a rate that the very meaning of Iwavelet analysis" keeps changing to incorporate new ideas Our paper contains some more recent developments and that it focuses on the multiresolution analysis" aspect of wavelets. JPEG image compression standard use DCT (DISCRETE COSINE TRANSFORM). The discrete cosine transform is a fast transform. It is a widely used and robust method for image compression. It has excellent compaction for highly correlated data.DCT has fixed basis images DCT gives good compromise between information packing ability and computational complexity. DWT can be used to reduce the image size without losing much of the resolutions computed and values less than a pre-specified threshold are discarded. Thus it reduces the amount of memory required to represent given image. Image compression is very important for efficient transmission and storage of images . Demand for communication of multimedia data through the telecommunications network and accessing the multimedia data through Internet is growing explosively. Image data comprise of a significant portion of the multimedia data and they occupy the major portion of the communication bandwidth for multimedia communication. The basic objective of image compression is to find an image representation in which pixels are less correlated. The two fundamental principles used in image compression are redundancy and irrelevancy. Redundancy removes redundancy from the signal source and irrelevancy omits pixel values which are not noticeable by human eye. JPEG. the need for image compression, consider the transmission of low resolution 512 × 512 x 8 bits/pixel x 3 color video image over telephone lines. Using a 96000 bauds(bits/sec) modem, the transmission would take approximately 11 minutes for just a single image, which is unacceptable for most applications.

JPEG provides a compression method that is capable of compressing continuous-tone image data with a pixel depth of 6 to 24 bits with reasonable speed and efficiency.JPEG may be adjusted to produce very small, compressed images that are of relatively poor quality in appearance but still suitable for many applicationsDCT separates images into parts of different frequencies where less important frequencies are discarded through quantization and important frequencies are used to retrieve the image during decompression. Compared to other input dependent transforms, DCT has many advantages: (1) It has been implemented in single integrated circuit; (2) It has the ability to pack most information in fewest coefficients; (3) It minimizes the block like appearance called blocking artifact that results when boundaries between sub-images become visible. The forward 2D_DCT transformation is given by the following equation:

$\mathrm{C}(\mathrm{u}, \mathrm{v})=\mathrm{D}(\mathrm{u}) \mathrm{D}(\mathrm{v}) \mathrm{f}(\mathrm{x}, \mathrm{y}) \cos [(2 \mathrm{x}+1) \mathrm{u} \pi / 2 \mathrm{~N}] \cos [(2 \mathrm{y}+1) \mathrm{v} \pi / 2 \mathrm{~N}]$

Where, u,v $=0,1,2,3, \ldots \ldots \ldots \ldots . ., \mathrm{N}-110 \mathrm{Nx} 10 \mathrm{Ny}$

where 
$\mathrm{D}(\mathrm{u})=(1 / \mathrm{N}) \wedge 1 / 2$ for $\mathrm{u}=0$

$\mathrm{D}(\mathrm{u})=2(/ \mathrm{N})^{\wedge} 1 / 2$

For

$\mathrm{u}=1,2,3 \ldots \ldots,(\mathrm{N}-1)$

Original image is divided into blocks of $8 \times 8$. Pixel values of a black and white image range from $0-255$ but DCT is designed to work on pixel values ranging from -128 to 127 .Therefore each block is modified to work in the range. Equation(1) is used to calculate DCT matrix. DCT is applied to each block by multiplying the modified block with DCT matrix on the left and transpose of DCT matrix on its right. Each block is then compressed through antization.Quantized matrix is then entropy encoded. Compressed image is reconstructed through reverse process. Then Inverse DCT is used for decompression

Discrete Wavelet Transformation (DWT) transforms discrete signal from the time domain into time frequency domain. The transformation product is set of coefficient organized in the way that enables not only spectrum analysis of the signal but also spectral behavior of the signal in time. The wavelet transform has emerged as a cutting edge technology, within the field of image compression. Waveletbased coding provides substantial improvements in picture quality at higher compression ratios . Over the past few years, a variety of powerful and sophisticated wavelet-based schemes for image compression have been developed and implemented

\section{DCT and wavelet based Image Coding}

Discrete cosine transform (DCT) is widely used in image processing, especially for compression. Some of the applications of two-dimensional DCT involve still image compression and compression of individual video frames.The JPEG standard has been an effective first solution to the standardization of image compression. JPEG has some very useful strategies for DCT quantization and compression, it was only developed for low compressions. The JPEG (Joint Photographic Experts Group) standard has been around for some time and is the only standard for lossy still image compression. There are quite a lot of interesting techniques used in the JPEG standard and it is important to give an overview of how JPEG works. When the Wavelet Transform coupled with the baseline JPEG quantizer, the wavelet coefficients are rearranged into wavelet blocks and scanned into vectors before scalar quantization and Huffman coding. DCT based embedded image coder. An 8x8 DCT image representation can be thought of as 64 sub band decomposition, and that we can treat each 8x8 DCT block as a depth-three tree of coefficients. The DCT based coder has lower complexity than its wavelet based counterpart. We compared the quality of JPEG compressed images against the quality of images compressed with a variety of wavelet filters, in terms of the SNR and the subjective image quality. We looked at 3 important classes of images: 4 natural images, 3 synthetic images and 4 textual images were used. The images were all 256 by 256 in size.

The discrete wavelet transform (DWT) is a mathematical tool that has aroused great interest in thefield of image processing due to its nice features. Someof these characteristics are:

1) it allows image multi resolution representation in anatural way because more wavelet sub bands are used to progressively enlarge the low frequency subbands;

2) It supports wavelet coefficients analysis in bothspace and frequency domains, thus the interpretation of the coefficients is not constrained to its frequency behavior and we can perform better analysis for image vision and segmentation; and

3) For natural images, the DWT achieves high compactness of energy in the lower frequency subbands, which is extremely useful in applications such as image compression. The introduction of the DWT made it possible to improve some specific applications of image processing by replacing theexisting tools with this new mathematical transform.

Wavelet transform (WT) represents an image as a sum of wavelet functions (wavelets) with different locations and scales. Any decomposition of an image intowavelets involves a pair of waveforms: one to representthehigh frequencies corresponding to the detailed partsof an image (wavelet function $\psi$ ) and one for the lowfrequencies or smooth parts of an image (scaling function Ø). DWT is a multi resolution decompositionscheme for input signals. The original signals are first

\subsection{Wavelet decomposition}

We should theoretically deal with data of infinite length in this setup. But in practice, let's assume the data has length $2 \mathrm{~N}$. The full wavelet decomposition is found via the pyramid, or tree, algorithm: The pyramid filtering is performed forN steps, and each step gives sequences half the size of the sequences in the previous step. The intermediate sequences obtained by filtering byHare called the scaling coefficients. The wavelet coefficients of the data then consist of all the sequences wl. In reality, the decomposition is truncated after a given number of steps and data length does not have to be a full power of 2 . The original data can now be reconstructed from the wavelet coefficients and the one final scaling coefficient sequence by using the 
reconstruction pyramid: this is the decomposition pyramid with the arrows reversed, and using the filters HT and GT.. The continuous wavelet transform for a given basic wavelet was defined as the function taking the scaling and translation parameters $\mathrm{a} ; \mathrm{b}$ to the inner product with the scaled and translated wavelet $\mathrm{a} ; \mathrm{b}$ : The wavelet transform is a map from the space of square integrable functions, L2, into the space of squareintegrable functions of two real variables, L2(R2). Typically, this map takes functions into a proper subset of L2(R2). This means that not all reasonable functions wf : $(a ; b) 7$ ! wf $(a ; b)$ are wavelet transforms ofany function and there are heavy constraints on such wf. This fact can be illustrated by using the Gaussian "wavelet".

\subsection{JPEG 2000 wavelet-based standard}

JPEG 2000 is a wavelet-based standard for the compression of still digital images. It was developed by the ISO JPEG committee to improve on the performance of JPEG while adding significant new features and capabilities to enable new imaging applications. The JPEG 2000 compression method is part of a multi-part standard that defines a compression architecture, file format family, client-server protocol and other components for advanced applications

These opportunities are enabled by the JPEG 2000 feature set:

- A single architecture for lossless and visually lossless image compression

- A single JPEG 2000 master image can supply multiple derivative images

- Progressive display, multi-resolution imaging and scalable image quality

- The ability to handle large and high-dynamic range images

- Generous metadata support

Low bit-rate compression performance

- Lossless and lossy compression in a single codestream.

- Large images, noting that JPEG was limited to $64 \mathrm{~K}$ by $64 \mathrm{~K}$ images without tiling.

- Single decompression architecture to integrate features and applications in a

unified framework

- Transmission in noisy environments

- Computer-generated imagery, which is smoother and less noisy compared to

natural imagery

- Compound documents, which combine images and text.

With JPEG 2000, an application can access and decode only as much of the compressed image as needed to perform the task at hand. This means a viewer, for example, can open a gigapixel image almost instantly by retrieving and decompressing a low resolution, display-sized image from the JPEG 2000 codestream. JPEG 2000 also improves a user's ability to interact with an image. The zoom, pan, and rotate operations that users increasingly expect in networked image systems are performed dynamically by accessing and decompressing just those parts of the JPEG 2000 codestream containing the compressed image data for the region of interest.

\section{Results and Performance}

We can start from the filters (hi) and (gi) and try to pin down the requirements needed for these to generatea valid orthonormal multiresolution and wavelet. It's convenient to look at these in Fourier transform form.
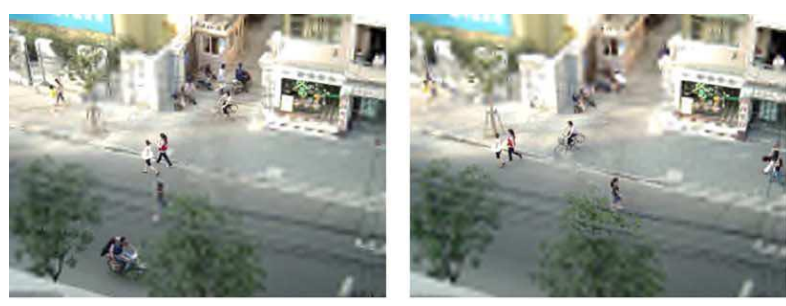

Fig a) Compressed results in jpeg format

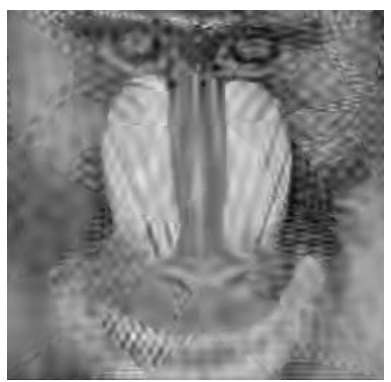


Fig a) DCT-level transforms

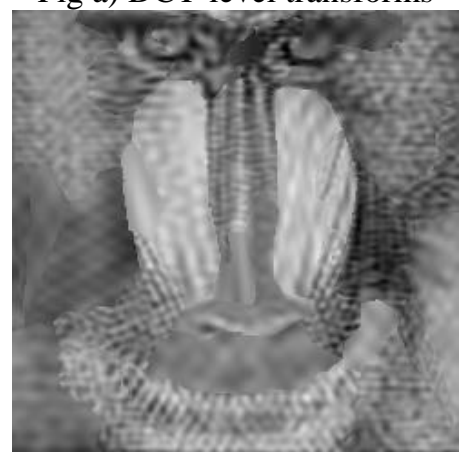

Fig B)JPEG standard

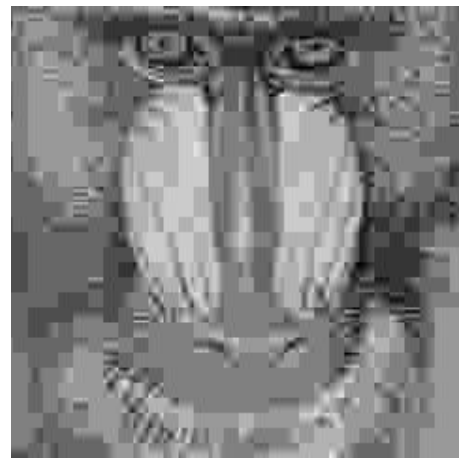

FIG C) JPEG 2000 standard

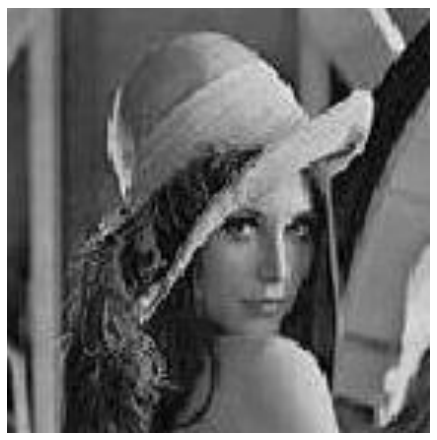

Fig(a) original image

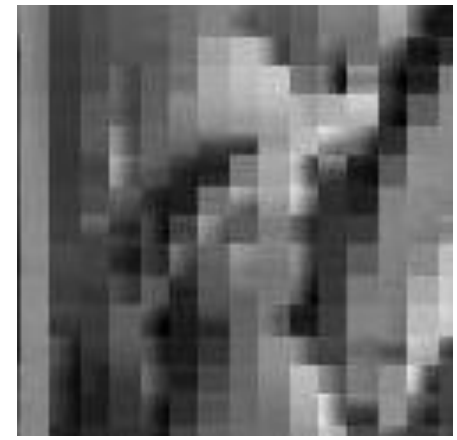

Fig (b) compressed image with DCT

Our approach is based on DCT and JPEG standard baseline coder results shown above.All of them have very similar PSNR performance even with JPEG but JPEG is affected by disturbing blocking effect . 


\section{Conclusion}

Our work deals with deals with the JPEG Encoder for image compression upon comparing with Discrete cosine transform (DCT) is widely used in image processing, especially for compression based on DCT and JPEG Standard coder and JPEG 2000 for image compression .

\section{References}

[1] “A Novel Multiresolution Spatiotemporal Saliency Detection Model and Its Applications in Image and Video Compression" IEEE TRANSACTIONS ON IMAGE PROCESSING, VOL. 19,

[2] GHANBARI, M. Video Coding: An Introduction to Standard Codecs. IEE, London, 1999, p. 60-62.

[3] G. Davis, "Wavelet Image Compression ConstructionKit,"http:/www.geoffdavis.net/dartmouth/wavelet/wavelet.html

[4] R. C. Gonzalez and R. E. Woods, "Digital Image Processing”,Second edition,pp. 411-514, 2004

[5.] N. Ahmed, T. Natarajan, and K. R. Rao, "Discrete cosine transform," IEEE Trans. on Computers, vol. C-23, pp. 90-93,1974.

[6.] A. S. Lewis and G. Knowles, "Image Compression Using the 2-D Wavelet Transform" IEEE Trans. on Image Processing, Vol. I . NO. 2, PP. 244 - 250, APRIL 1992.

[7] M. Antonini , M. Barlaud and I. Daubechies, "Image Coding using Wavelet Transform",IEEE Trans. On Image Processing Vol.1, No.2, pp. 205 - 220, APRIL 1992.

[8] S. Mallat, "A Theory for Multiresolution Signal Decomposition: The Wavelet Representation," IEEE Trans. on Pattern Analysis and Machine Intelligence, Vol. 11, No.7, pp. 674-693

\section{Author's Information}

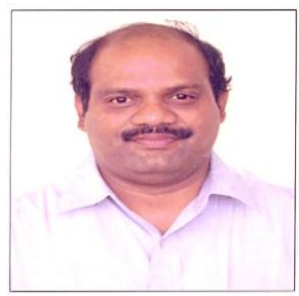

1.Ravi Mathey is a post-graduate specialized in Computer Science from BIT -Ranchi and he did Instrumentation technology in Andhra university. He has more than 18 years of experience in Research and Development, Presently he is working as a Associate Professor and HOD of CSE Department at Vidya Jyothi Institute of Technology (VJIT). His area of research is wireless embedded application and image compression techniques by using fractals and wavelets.

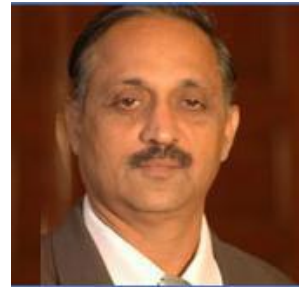

2.Prof. P.S.Avadhani did his Masters Degree and PhD from IIT, Kanpur. He is presently working as Professor in Dept. of Computer Science and Systems Engineering in Andhra University college of Engg., in Visakhapatnam. He has more than 50 papers published in various National / International journals and conferences. His research areas include Cryptography, Data Security, Algorithms, and Computer Graphics, Digital Forensics 